\title{
Editorial
}

\section{Functional Biomarkers for Alzheimer's Disease}

Alzheimer's disease (AD) has seen a tremendous growth of candidate biomarkers in the last decade. The role of such established or research biomarkers is to allow AD to be diagnosed with high accuracy, to infer about its prognosis, monitor disease progression and evaluate changes induced by 'disease-modifying' drugs. An ideal biomarker should detect a specific pathophysiological feature of $\mathrm{AD}$, thus not present under the healthy condition, in other primary dementias, or in confounding conditions. Besides being reliable, a biomarkers should be relatively non-invasive, simple to perform, widely available and not too expensive. At present, no candidate meets these requisites, that represent the high standards to which the research field aspires.

Genetic markers have been recognized for many years in the genetic forms of AD, and there are now multiple known mutations across three separate chromosomes with others likely to be identified in the future. Strictly speaking, these specific mutations are long-term prognostic biomarkers of AD and not diagnostic biomarkers. While not a mutation, APOE $\varepsilon 4$ (chromosome 19) is the most popular genetic marker for sporadic, multifactorial AD. Combining the risk associated with the APOE $\varepsilon 4$ allele with neuroimaging measures has produced a potential for further refinement of identifying individuals at greater risk for developing AD. Plasmatic factors, including $\beta$-amyloid, are very attractive as potential biomarkers but there is much question concerning the variability of the plasma levels and whether they are significantly related to brain levels and the disease process itself. The specificity of these biomarkers is not yet well established versus other forms of dementia. On the other hand, a relevant bulk of information is now available on some CSF biomarkers, notably the reduced levels of A $\beta$ 1-42 and increased levels of Tau protein and its phosphotilated form, but further CSF biomarkers are under active investigation. Various morphological brain measures by means of MRI, ranging from total brain volume to hippocampal volume, have been proposed. The efforts nowadays are directed toward finding an automated, unsupervised method to detect atrophy in a specific brain region, such as the Medial Temporal Lobe. Functional neuroimaging with SPECT and especially with FDG-PET has repeatedly shown brain dysfunction in the associative neocortical areas, starting from the posterior parietal and cingulate cortex and extending to the more anterior regions. Especially with concurrent cognitive testing, SPECT and PET have yielded rather accurate diagnostic figures. However, standard operational procedures on how to treat these functional images are still lacking. A more recent and exciting development has been the markers for measuring the brain $\beta$-amyloid1-42 or neurofibrillary tangle burden. The high percentage of positive scans in apparently normal aged subjects has raised concerns about its specificity, and it is likely a longterm clinical follow-up of these subjects is needed before their diagnostic use can be spread. Finally, EEG is an old tool of modest utility in its classical form, but recent post-processing algorithms seem to have re-vitalized it, especially when looking at the temporal span which is its main attractive characteristic.

As noted previously, it is imperative to compare the biomarkers in AD patients against other related diseases to investigate whether or not the measures are diagnostic for $\mathrm{AD}$ or non-specific but sensitive markers of a general neurodegenerative process. To date, the possibility of combining risk factors from various modalities (i.e., genetic, CSF and plasma, neuropsychological testing, morphological and functional neuroimaging studies) offers a golden opportunity to maximize the individual risk factors and predict $\mathrm{AD}$ at a MCI stage or even earlier. Disease-modifying drugs are hopefully coming and the need to diagnose $\mathrm{AD}$ with high accuracy has become stringent.

\section{PAPERS AND AUTHORS}

\section{$>\quad$ Genetic and Blood Biomarkers}

Parastoo Momeni and Raffaele Ferrari

$>\quad$ CSF Biomarkers

Alessandra Piccini

$>\quad$ Automatic Morphological Analysis of Medial Temporal Lobe Andrea Chincarini, Mirko Corosu, Gianluca Gemme, Piero Calvini, Roberta Monge, Maria Antonietta Penco, Luca Rei, Sandro Squarcia, Patrizia Boccacci and Guido Rodriguez

Is there Still a Place for Perfusion SPECT in the Diagnosis of Dementia?

Klaus P. Ebmeier

FDG-PET

Flavio Nobili and Silvia Morbelli

$>\quad$ Amyloid PET

Alexander Dredzga and Robert Perneczky 
$>\quad$ Quantitative Electroencephalography

Claudio Babiloni, Fabrizio Vecchio, Roberta Lizio, Paolo M Rossini, Giovanni B. Frisoni, Raffaele Ferri and Guido Rodriguez

$>\quad$ How to Distinguish Alzheimer's from Lewy-Body Dementia?

Ugo Paolo Guerra and Pietro Tiraboschi

Flavio Nobili

(Guest Editor)

Department of Neuroscience

Ophthalmology and Genetics

Azienda Ospedaliera-Universitaria S. Martino

Genoa

Italy

E-mail: flaviomariano.nobili@hsanmartino.it

This is an open access article licensed under the terms of the Creative Commons Attribution Non-Commercial License (http: //creativecommons.org/licenses/by-nc/ 3.0/) which permits unrestricted, non-commercial use, distribution and reproduction in any medium, provided the work is properly cited. 